\title{
A SOFTWARE FRAMEWORK FOR DYNAMIC MODELING OF DC MOTORS AT ROBOT JOINTS
}

\author{
Mehmet Serdar Güzel ${ }^{1}$ \\ ${ }^{1}$ Ankara University, Computer Engineering Department, Ankara, Turkey
}

\begin{abstract}
This paper addresses to develop an educational simulation tool for dynamic analysis of a 5 DOF robot arm's actuators. Kinematics analysis of robot arm was visually displayed with the corresponding tool in relation to dc motors, used at the robot arm joints. Besides, dynamic modeling of DC motors is presented and their combined motion within the arm is analyzed and displayed visually.
\end{abstract}

Keywords: Robot arm, Robot Kinematics, PWM, DC Motor Simulation, Robot Simulator.

$* * *$

\section{INTRODUCTION}

Simulation is an engineering tool, used in designing and analyzing of real time systems. It is mainly used to provide a safe, reliable and inexpensive way for the operation of complex systems. By using a simulator, experimental designs can be effectively validated without consuming materials and machining efforts in the workshop $[1,2,3]$.

This paper presents the simulation of a position control system using robot arm actuators having Five Degree of Freedom (DOF). The simulation was mainly designed and implemented based on the general mathematical model of DC servo motors. Lynx-6 robot arm with 5 DOF was modelled analytically and its forward and inverse kinematics was analyzed and calculated. Consequently, a final model was generated and a real time simulation was performed based on the proposed model. Lynx-6 robot arm which is shown in figure 1 has 5 DOF with a grip movement. It is similar to human arm from the number of joints point of view. These joints produce shoulder rotation, shoulder back and forth, elbow, wrist up and down, wrist rotation and gripper motion. [4,5]. One of the most challenging and critical problems in control engineering is to control analog voltages with digital inputs. PWM (PulseWidth Modulation) technique is used to vary the analogue voltages by using digital pulses; these digital pulses are generated through computer programming according to motion specifications of the user by a servo control card. Control cards are utilized to handle the given problem. In this study, a SSC-32 servo control card has been attached into the Lynx-6 Robot arm. The card mainly aims to convert the digital pulses to dc voltages during the pulse duration to drive the dc motors of the joints in required amounts [6]. In the application part of this study a high level visual software package was developed to simulate dynamically both a Lynx6 Robot arm's actuators and their combined motion .Visual Studio Net platform with C\# programming Language was employed to developed this visual software package.

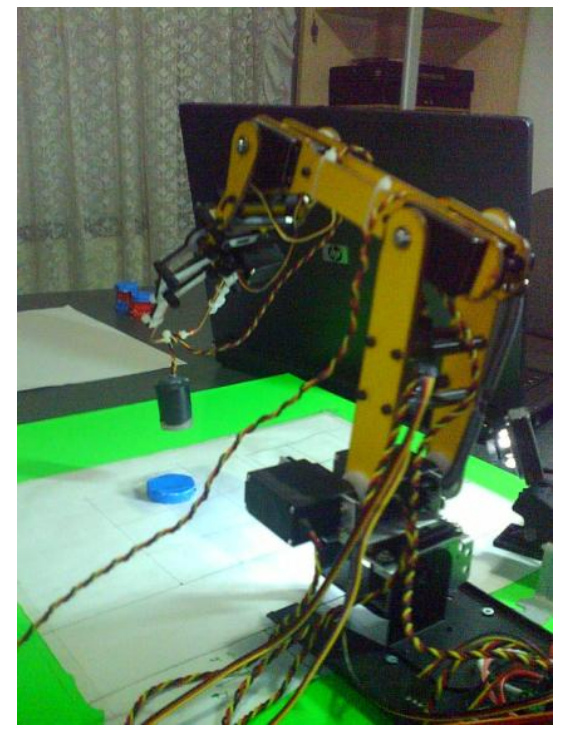

Fig 1: Lynx-6 Robot Arm

\section{DC MOTOR SIMULATION}

DC motors are preferred to be used in control systems due to the fact that they provide continuous motion unlike the stepper motor motion in steps. Mathematical model of a DC motor is derived to observe and define the control mechanism analytically. The most common control technique for DC motors is the armature-current control to generate rotational motion. [7]. A typical DC motor, used in the Lynx-6 robot arm is illustrated in Figure 2. 


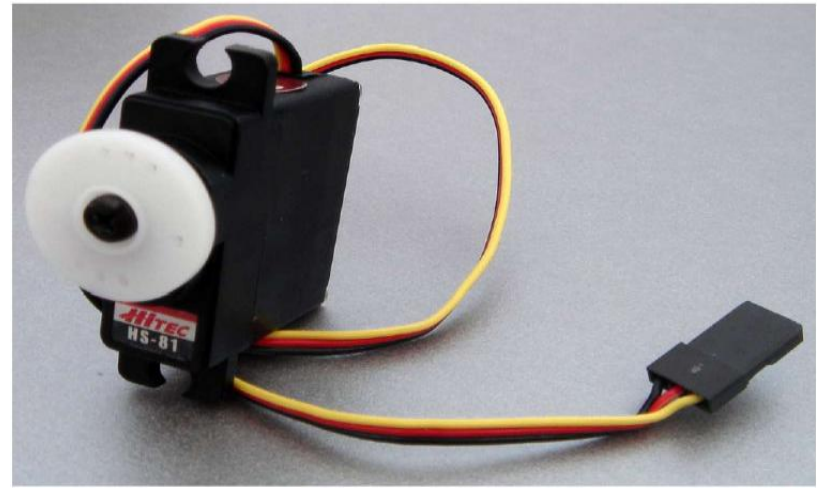

Fig 2: DC Motor

The basic mathematical model of a DC motor, employed in the corresponding robot arm, was shown in Figure 3. In the model, $\boldsymbol{v}_{\mathrm{a}}$ is the supply voltage, $\boldsymbol{i}_{\mathrm{a}}$ is the winding current, $\mathbf{R}_{\mathrm{a}}$ is the resistance of the armature circuit, $\mathbf{L}_{\mathbf{a}}$ is the inductance of the armature circuit, $\mathbf{J}$ is the moment of inertia of the system (including the motor and the load), the back emf is $\mathbf{e}_{\mathbf{a}}=$ $\mathbf{k}_{\mathrm{e}} \cdot \boldsymbol{\omega}_{\mathrm{a}}$ (here $\mathrm{k}_{\mathrm{e}}$ is known as the back emf constant or torque constant $\boldsymbol{\omega}_{\mathbf{a}}$ is the angular speed of the system, $\mathbf{T}_{\mathbf{e}}$ is the electromagnetic torque developed by the motor, $\mathbf{T}_{\mathbf{L}}$ is the load torque, and $\mathbf{B}$ is the damping coefficient which is usually ignored. The comprehensive information about these is given in reference [8].

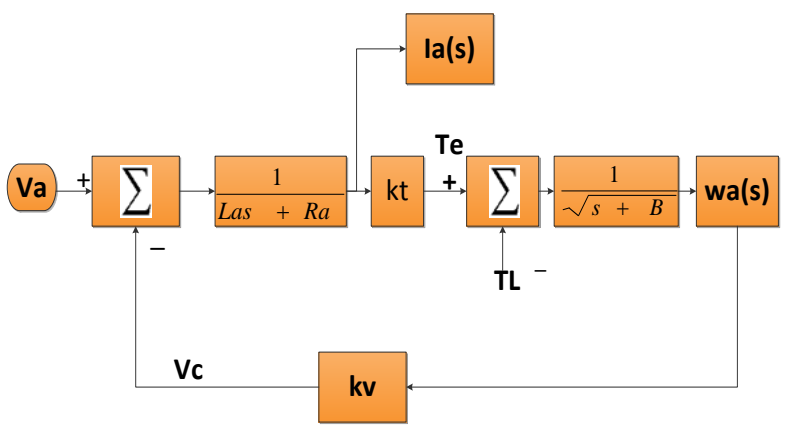

Fig 3: Mathematical model of a DC Motor

The dynamic simulation of the dc motor constitutes:

a) voltage/current equation of the motor armature,

b) Dynamic equation of the motor-load (mechanical) system,

c) Electromagnetic torque equation

$$
\begin{aligned}
& v_{a}=R_{a} i_{a}+L_{a} \frac{d i_{a}}{d t}+e_{a} \\
& T_{e}=J \frac{d \omega_{m}}{d t}+B \omega_{m}+T_{L}
\end{aligned}
$$

$$
T_{L=} \mathrm{k}_{\mathrm{e}} \mathrm{i}_{\mathrm{a}}
$$

The general formula to solve differential equations given in (2) and (3) is illustrated as follows :

$$
x(t)=x(\infty)+[x(t o)-x(\infty)] e^{(t-t o) / t c}
$$

Where (tc) time constant for RL circuits is $\mathrm{L} / \mathrm{R}$, Equation 4 can be interpreted as in equation (5) due to fact that all Resistors, Capacitors, and Inductors have positive values when $\mathrm{t}>0$ [7].

\section{PWM (PULSE-WIDTH MODULATION)}

PWM was employed in variety of applications, ranging from measurement and communications to power control. It is a way of digitally encoding analog signal levels. Through the use of high-resolution counters, the duty cycle of a square wave is modulated to encode a specific analog signal level. At any given time, the full DC supply is either fully ON or fully OFF.

The voltage or current source is supplied to the load by means of a series of on and off pulses. The on-time is the time during which the DC supply is applied to the load, and the off-time is the period during which the supply is switched off. Figure 4 presents three different PWM signal outputs, used in actuator motor control. For example PWM signal output with $\% 10$ duty cycle in (a) is ON during \%10 of its period and OFF during $\% 90$ of its period. Others in (b) and (c) have the similar properties with different ON/OFF periods.

The initial position of any dc servo motor of Lynx-6 robot arm was compared with the new required positions which were both defined by user program parameters. The difference between positions is applied as an input signal to servo card and the essential PWM signal was generated to feed the dc motors with dc supply. Robot arm motors were chosen to be used in no more than 90 degree range of motion. Hence the corres- ponding "width" of the PWM signal pulse varied from 0.9 milliseconds (minimum position), to $2.1 \mathrm{~ms}$ (maximum position). The Pulse is repeated every 14 to 20 milliseconds [9] .

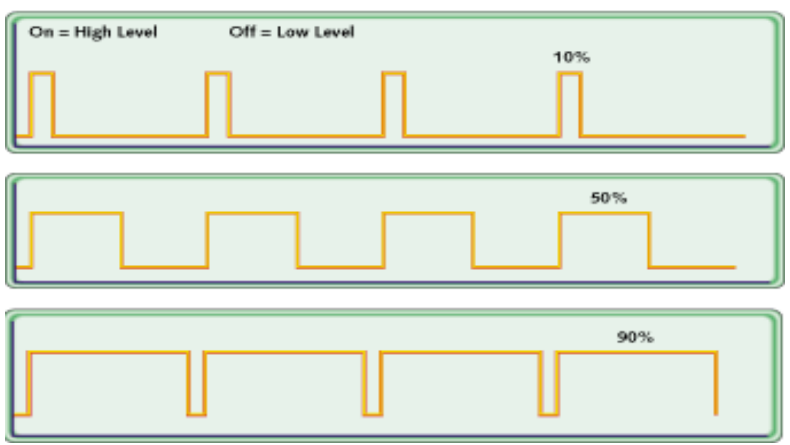

Fig 4: PWM signals with varying Duty Cycles a) $\% 10$ duty cycle b) $\% 50$ duty cycle c) $\% 90$ duty cycle 


\section{KINEMATICS}

Denavit-Hartenberg representation is used to model the joints of Lynx-6 Robot arm, [10] , can be seen in Figure 5. All the joints are assigned by using the principles of $\mathrm{D}-\mathrm{H}$ convention.

Robot kinematics is mainly divided into two groups, namely, forward kinematics and inverse kinematics. In forward kinematics, the length of each link and the angle of each joint are given and the position of any point in the work space of the robot is calculated. As opposed to forward kinematics, in inverse kinematics, the length of each link and the position of the point in work space are given and the angle of each joint is calculated.

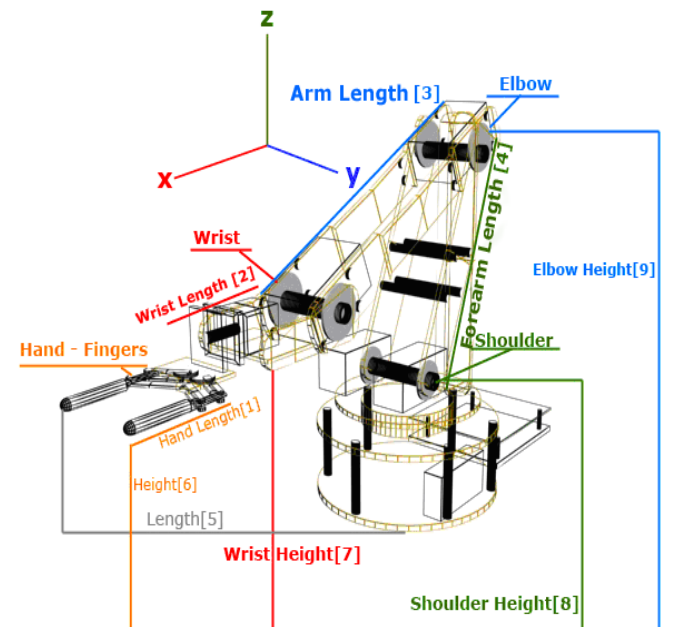

Fig 5: Model of Lynx-6 Robot Arm

\subsection{Forward Kinematics}

To calculate forward kinematics equations for 5 dof Lynx arms, the transformation matrices corresponding to robot arm joints, [11], were manipulated and the final forward kinematics solution was shown below.

$$
\mathrm{T}_{\mathrm{f}=\mathrm{A}_{1}} * \mathrm{~A}_{2} * \mathrm{~A}_{3} * \mathrm{~A}_{4} * \mathrm{~A}_{5} \text {, for } 5 \text { Dof }
$$

\subsection{Inverse Kinematics}

Inverse Kinematics analysis determines the joint angles for desired position and orientation in Cartesian space. The robot arm manipulator which is used in this study has five degrees of freedom (DOF). In order to determine the joint angles, the final matrix equation is multiplied by $\mathrm{A}_{\mathrm{n}}^{-1} \quad(\mathrm{n}=1,2,3,4,5,6)$ on both sides sequentially and the generated linear equations were solved [11]. The inverse kinematic solutions for five DOF Lynx arm are shown below:

$$
T_{f}=\left[\begin{array}{cccc}
n x & o x & a x & p x \\
n y & o y & a y & p y \\
n z & O z & a z & p z \\
\mathrm{O} & \mathrm{O} & \mathrm{O} & 1
\end{array}\right]
$$

$\theta_{234}$ (WARTG=Wrist Angle Relative to $\mathrm{G}$ round)

$$
\begin{gathered}
\theta 2=\arctan (\mathrm{S} 2 / \mathrm{C} 2) \\
\theta 3=\arctan (\mathrm{S} 3 / \mathrm{C} 3) \\
\theta 4=\theta 234-\theta 3-\theta 4 \\
\theta 5=\arctan (\mathrm{S} 5 / \mathrm{C} 5)
\end{gathered}
$$$$
\theta 1=\arctan (\mathrm{py} / \mathrm{px}) \text { or } \theta 1=\theta 1+180^{\circ}
$$

\section{SOFTWARE DESIGN}

The control program has been implemented using $\mathrm{CH}$ programming language in Visual Studio Net platform. The program supports two main operation modes, simulation of DC servo motors and kinematics analysis of Lynx-6. Main Screen of the program was shown in figure 6 .

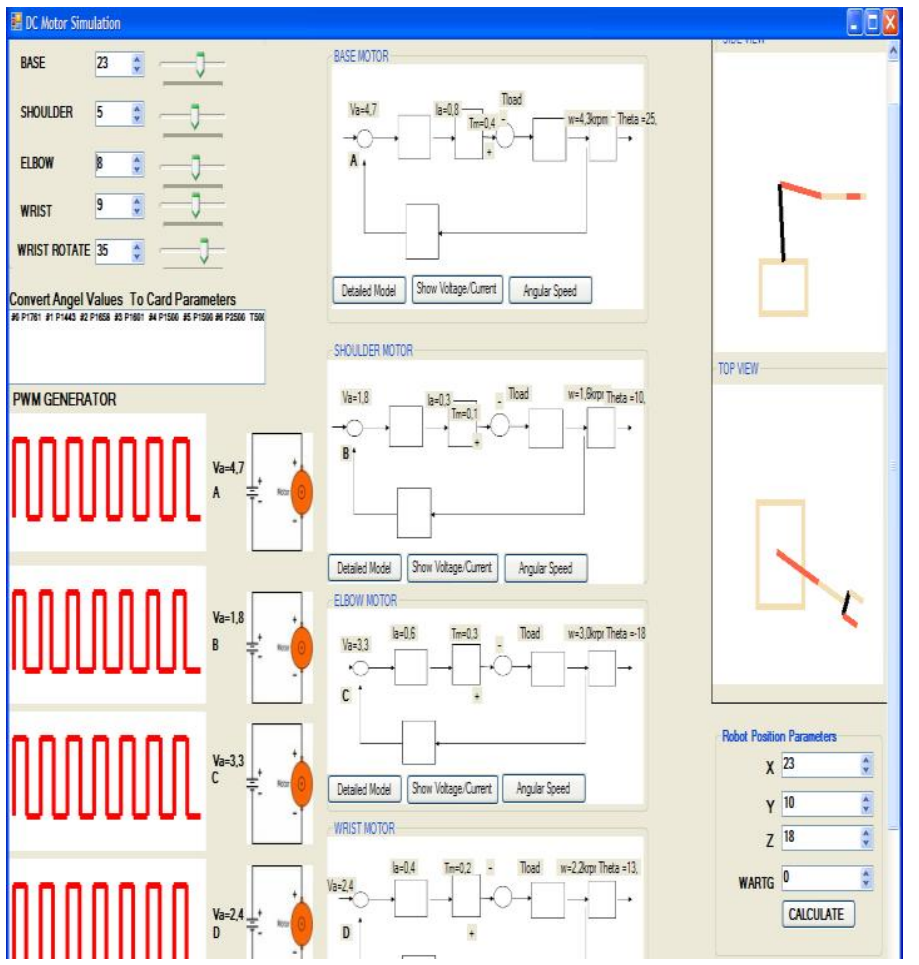

Fig 6: Main Screen of the Framework 
On the left side of the main screen, there are two graphical views, side view and top view of the robot arm, are displayed to show the robot arm's motion in real time. There are three engines exist within the software package, namely, Kinematics Engine, PWM Engine, and Simulator Engine.

Kinematics Engine: It is responsible with the solutions of forward and inverse kinematics equations and the calculations related to joints and Cartesian coordinates.

PWM Engine: It is responsible with the managing of analogue voltages' and digital pulses and provides correlation between user defined input values (angle or position) and control card parameters.

Simulator Engine: It is responsible with the interpretation of the robot model, construction of internal data structures to represent the robot and manipulating the joint angles, as specified by simulation commands and the robot's kinematics

The developed software is mainly designed to simulate and show the operational stages of the robot arm. The general flowchart is shown in figure 7.

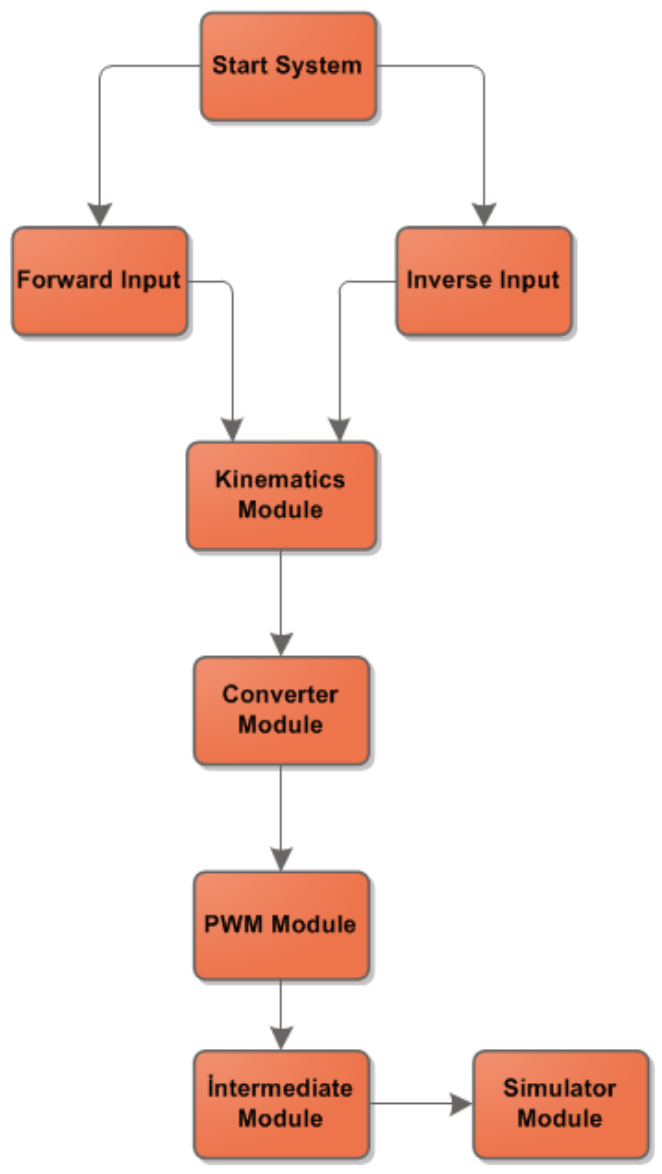

Fig 7: Flow Chart Diagram of the Software
The steps of algorithm are defined as follows:

a) Program is started.

b) Data is entered either as degree angles from Forward Kinematics Input Screen or as position coordinates from Inverse Kinematics Input Screen.

c) If given input values are position values; Kinematics Engine start to work and use inverse kinematics equations to derive required input angle values, which has been detailed in Kinematics section.

d) Derived angle values must be converted into control card (SSC-32) parameters [6].

e) PWM engine is responsible for managing analogue values with digital inputs, using applied voltage Interval, paired with corresponding angle value by the engine.

f) All related joints engines are start to work for dynamic simulation with given constant motor parameters such as Armature Resistance, Inductance etc. and calculated values as applied voltage by PWM engine.

g) Simulator Engine starts to show the work. All motional characteristics of robot arm were displayed with these models.

There are also graphical windows on the main screen for all joints to show robot joint response such as current vs time (current responses to varying time) and angular speed vs time (speed response to varying time) graphs. An example of robot base joint is shown in figures 8 and 9 respectively.

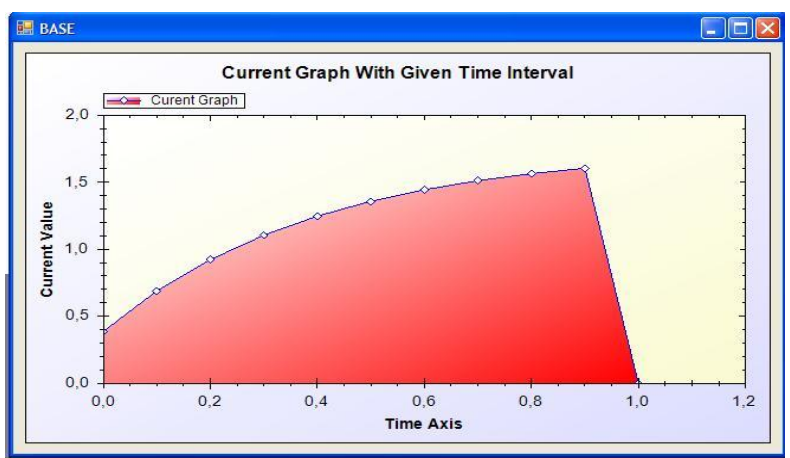

Fig 8: Current-Time Graph for Base (45 degrees)

Figure 8 presents an experiment in which it can be inferred that as 2.7 volt is applied to the system; the motor can only achieve a maximum value of 1.8 amber. Besides, it takes the motor one second to reach its steady-state value. This example in essence shows the change in current according to the time interval. Additionally, Angular Speed/Time graph of given software is also shown below that as the same volt is applied to the system, the motor can only achieve a maximum speed of $5 \mathrm{rad} / \mathrm{sec}$, which also takes the motor one second to reach its steady-state speed. 


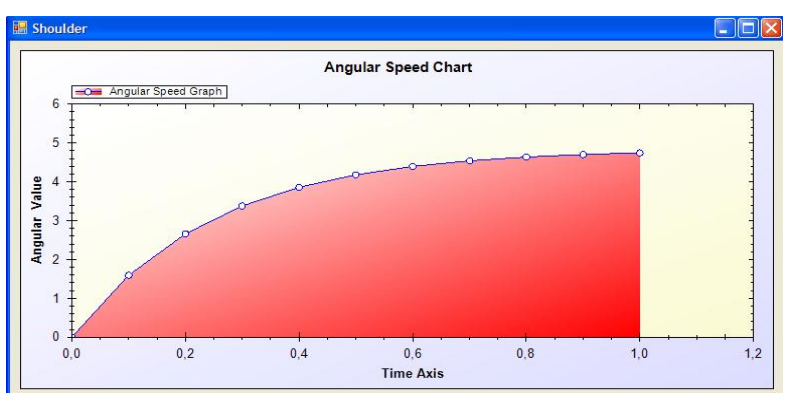

Fig 9: Angular Speed-Time Graph for Base (45 degrees)

There is also a kinematics screen which shows all transformation matrices which are derived from Final Kinematics Matrices is shown in figure 10. This screen is developed for detailed analysis of Kinematics equations. All transformation matrices of related joints can be observed and tested by users.

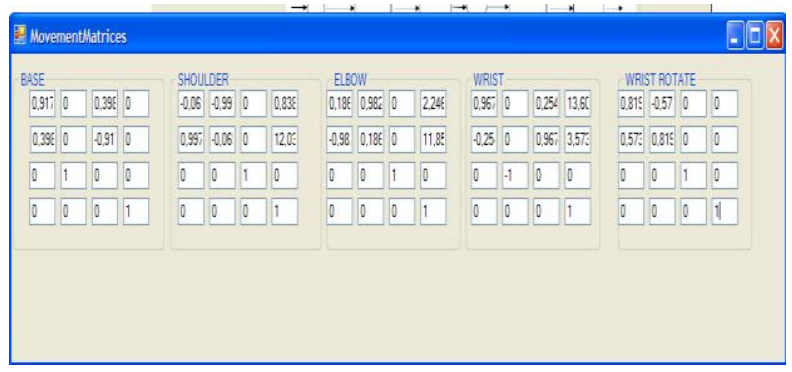

Fig 10: Movement Matrices for $(x=23 ; y=10 ; z=23)$

\section{DISCUSSION AND CONCLUSIONS}

This section addresses to discuss the results of a numerical example that all calculation steps including the simulation logic of the proposed software framework will be detailed as follows:

The following initial values are given for the experiment:

$\mathrm{Ra}=5$; Armeture Resistance

$\mathrm{L}=2$; Inductance

$\mathrm{Jm}=0.025$; motor constant

$\mathrm{Bm}=0.1$; friction constant

$\mathrm{kb}=0.1$; back emf constant

$\mathrm{Ki}=0.5$; moment constant

$\mathrm{T}_{1}=0.0$; Load moment

for given $0-6 \mathrm{~V}$ applied voltage interval

Position $\rightarrow$ Angle Values are calculated

\begin{tabular}{|l|l|l|l|l|l|l|}
\hline $\mathrm{X}$ & $\mathrm{Y}$ & $\mathrm{Z}$ & BASE & SHOULDER & ELBOW & WRIST \\
\hline 23 & 8 & 21 & 20 & 9 & -3 & 35 \\
\hline
\end{tabular}

As angle values are calculated from position vector by Kinematics Engine into signals, they are ready to be used by the control card [6]. The signal consists of positive going pulses ranging from 0.5 to $2.5 \mathrm{mS}$ (milliseconds) long, repeated 50 times a second (every $20 \mathrm{mS}$ ). The servo positions its output shaft in proportion to the width of the pulse. PWM engine calculates signal values for four different joints simultaneously that applied voltages are also calculated by given pulse values.

Calculated pulse values are,
BAS
$1713 \mathrm{~ms}$
HOULDER ELBOW
WRIST

Calculated voltage values are,
BASE
SHOULDER ELBOW
$4.1 \mathrm{~V}$
$2.5 \mathrm{~V}$
$1.4 \mathrm{~V}$
WRIST
$0.8 \mathrm{~V}$

Base motor simulator screen is illustrated in figure 11 and the corresponding Current-Time graph can also be seen in figure 12.

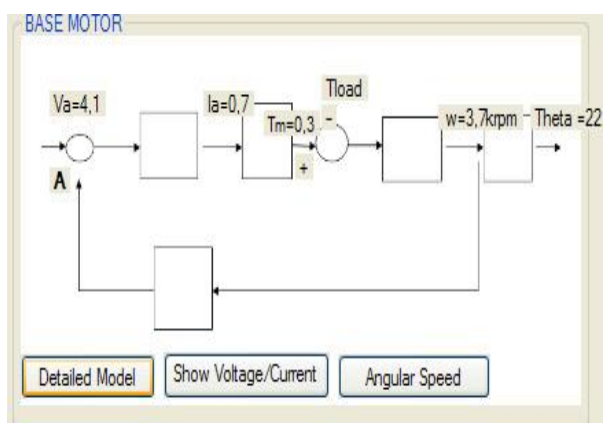

Fig 11: DC simulator for Base (22 degrees)

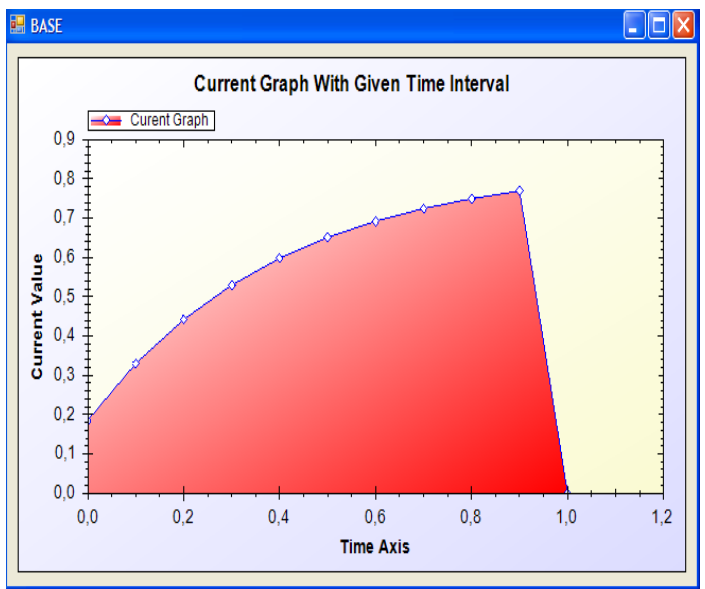

Fig 12: Current-Time Graph for Base (22 degrees)

At the final stage, the final position matrices are displayed and the simulator takes its new position with respected to the calculated angle values. 

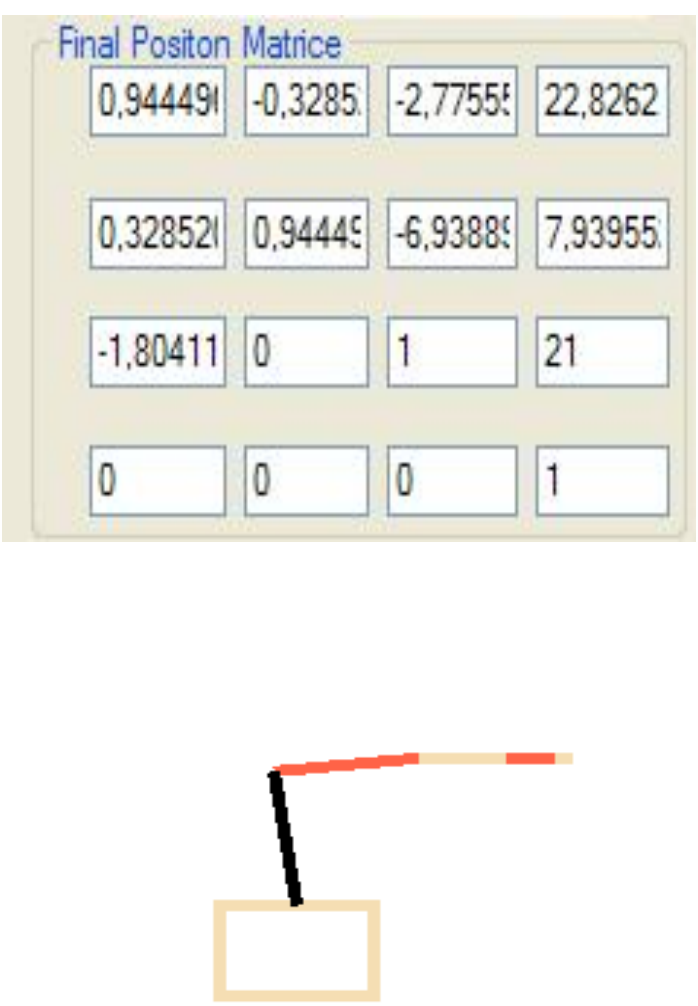

Fig 13: Final Position Matrices and Simulator

Overall, a new software tool for manipulator based simulation has been presented in this paper. The tool supports low cost Lynx arms which are mainly employed for scientific and educational applications. The tool analyzes manipulator actuators (DC servo motors), as well as it works on motional characteristics of manipulator and to be able to solve both inverse and forward kinematics. The proposed tool is flexible and open source that researches and students can use this tool for different tasks with minor modification. The interface of the proposed software framework is user friendly that researchers can learn and utilize the tool for their problems with minor effort.

The most powerful side of the tool is its ability to provide association between dynamic analysis of DC motors and Motional Characteristics of Lynx-6 robot arms. There exists more powerful DC motor simulators such as LABVIEW and MATLAB, but their costs are high for many researchers to handle and they do not provide association between dynamic analysis of DC motors and Kinematics of Lynx-6 Manipulators as the proposed software does.

Furthermore, the proposed software framework is free Software package and will be on the official web page of Computer Engineering Department of Ankara University (http://comp.eng.ankara.edu.tr/proje.html. This paper is a revised version of a conference paper that appeared as [12].

\section{REFERENCES}

[1]. White, R.B., Read, R., Koch, M. and Schilling, R.J., "A graphics simulator for a robotic arm," IEEE Trans. Education, Vol. 32, No. 4, pp. 417-429, (1989)

[2]. T. Raz, "Graphics robot simulator for teaching introductory robotics," IEEE Trans. Educ., vol. 32, pp. 153159, May (1989)

[3]. Antonia Yordan-Nones, Heterogeneous Modeling \& Design of Robot Arm Control System, , University of Puertro Rico, Mayagüez.

[4]. Koyuncu, B. and Güzel, M., Software Analysis of Lynx-6 Robot arm, ARC 2008, pp. 1-8

[5]. Güzel, M. S, Hinislioglu, Y., "A robotic software for intelligent applications, Artifical Life and Robotics 18 (1-2), 76-82

[6]. Lynx SSC-32 Servo motor controller board user manual. Available online at www.lynx.com. com. Accessed on December 2007

[7]. Luca Zaccarian , DC motors: dynamic model and control techniques , pp 16-22

[8]. Raymond A. DECARLO, Linear Circuit Analysis. pp. $224-229$, Prentice HALL.

[9]. Lynxmotion Inc ,2007 http://www.lynxmotion.com [10]. J. Denavit and R.S. Hartanberg, "A kinematic notation for lower-pair mechanisms based upon matrices" ASME J. Appl .Mechanics vol. 22, pp. 215 -221, 1955.

[11]. Koyuncu, B., and Güzel, M., "Software Development for the Kinematic Analysis of a Lynx 6 Robot Arm ,International Journal of Applied Science, Engineering and Technology , Volume 4, Number 4 pp. 228-233,2007

[12]. Koyuncu,B.,Güzel, M: A visual Tool for Kinematics Analyses of Lynx-6 Robot Arms together with dynamic modeling of dc motors at robot joints ,International conference on Automation, Robotics and control systems, arcs-08, Florida, USA,July 2008, pp 145-150. 